\title{
Surgical treatment by electrical stimulation of the auditory cortex for intractable tinnitus
}

\author{
C. Fabien Litré, $M D^{a}$, Etienne Theret, $M D^{a}$, Hugo Tran, $M D^{b}$, Marianne Lévèque, $M D^{b}$, \\ Christophe Portefaix, MD $^{c}$, Fabien Gierski, MD $^{c}$, Samuel Emeriau ${ }^{c}$, \\ Philippe Peruzzi, MD
}

\author{
${ }^{a}$ Department of Neurosurgery, Pr Rousseaux CHU Maison Blanche, Reims, France \\ ${ }^{b}$ Department of ENT, Pr Chays CHU Robert Debré, Reims, France \\ ${ }^{c}$ Department of Neuroradiology Pr Pierot CHU Maison Blanche, Reims, France
}

\begin{abstract}
Tinnitus is a public health issue in France. Around 1\% of the population is affected and 30,000 people are handicapped in their daily life. The treatments available for disabling tinnitus have until now been disappointing. We are reporting on the surgical treatment by electrical stimulation of the auditory cortex of a female patient affected by disabling tinnitus that resisted classical treatments. The tinnitus appeared suddenly 10 years ago after a left ear tympanoplasty. The acouphenometry measures revealed a bilateral tinnitus, predominant on the right side, constant, with high frequency $(6000 \mathrm{~Hz})$. Transcranial magnetic stimulation (TMS) was performed at first with several supraliminal and infraliminal protocols. This showed promising results. Anatomic and functional magnetic resonance imaging (fMRI) of the auditory cortex before and after repetitive TMS (rTMS) demonstrated a modification of the cortical activity and where the ideal location for a cortical electrode might be, to straddle primary and secondary auditory cortex. After these investigations, two quadra polar electrodes (Resume, Medtronic Ltd, Hertfordshire, UK), connected to a stimulating device implanted under the skin (Synergy, Medtronic Ltd), were extradurally implanted. The surgical procedure was similar to the one performed for analgesic cortical stimulation. No surgical complications were reported. The activation of the stimulator provided a reduction of $65 \%$ of the tinnitus impact, with a persistent effect on the right side. The feasibility of the cortical stimulation in symptomatic treatment of tinnitus was proven by this preparatory work. The middle- and long-term therapeutic effects remain to be evaluated.
\end{abstract}

(C) 2009 Elsevier Inc. All rights reserved.

Keywords tinnitus; auditive cortex; electric stimulation; transcranial magnetic stimulation

Tinnitus is a public health issue in France, with around $1 \%$ of the population affected and 30,000 people handicapped in their daily life.

Correspondence: Dr. Fabien Litre, Department of Neurosurgery, Maison Blanche, 45, Rue Cognacq Jay, Reims 51100, France.

E-mail address: fabien.litre@mac.com

Submitted July 17, 2008; revised November 8, 2008. Accepted for publication November 13, 2008.
Available treatments have been disappointing for disabling tinnitus. The treatment is delicate because of the psychological and behavioral repercussions of tinnitus, which leads many physicians to wonder about the organic origin of tinnitus. However, several neuroscience case studies have demonstrated the credibility of this symptom as a peculiar entity. In particular, the relationship between tinnitus and cerebral metabolic activity. ${ }^{1}$ 
The object of this work is to evaluate the feasibility of a neurosurgical treatment of tinnitus via an auditory cortex stimulation by a cortical electrode.

\section{Case report}

The patient was a 47-year-old right-handed woman. Her medical history shows several tympanoplasties 12 years ago for bilateral cholesteatoma (three surgeries on the left ear, and one on the right ear). She had depression for 8 years as a result of the tinnitus and has been unemployed since the permanence of her symptoms.

A bilateral tinnitus predominant on the right side suddenly appeared after the second left ear tympanoplasty. She received many medicinal treatments without any results (antiepileptic, antidepressant, vasodilator). At the time we first encountered the patient, she was taking benzodiazepine (clonazepam). She was motivated and willing to try this new therapy.

Audiometry showed bilateral hypoacusis, with bilateral mixed hearing loss, about $60 \mathrm{~dB}$ on the right side and $35 \mathrm{~dB}$ on the left side. The study of the tinnitus showed a bilateral tinnitus predominant on the right side $(70 \%$ on the right side, $30 \%$ on the left side) with high frequency $(6000 \mathrm{~Hz})$. It was only pure tone and no white-noise component. The intensity was important, assessed at $105 \mathrm{~dB}$ on the right ear. The tinnitus is a constant and permanent type. Three consultations, before surgery, with acouphenometry showed the same results.

"Tinnitus reaction questionnaire" is a validated questionnaire (TRQ, often called DET). Created by Wilson et $\mathrm{al}^{2}{ }^{2}$ it evaluates the psychologic distress caused by tinnitus. There are 26 questions and the scale has 5 points. The patient must reply to all the questions. We add up the points of the 26 questions to obtain the final score. Before surgery, the patient had a final score of 74 (severe tinnitus impact).

A visual analog scale (VAS)-type of measure was not used to gauge the patient's subjective experience. This type of measure is used for pain control after surgery. The use of the TRQ for tinnitus is preferred because it has a specific scale.

A consultation with a psychiatrist excluded any contraindications to the procedure, in particular the neurosurgical procedure, the under skin device, and the long-term follow-up.

\section{Procedure care}

This patient was integrated into our TMS $\mathrm{xx}$ tinnitus protocol. She had a course of 4 rTMS before the operation.

\section{rTMS procedure}

The patient has had four sessions of stimulation in 2 years, with several different protocols. Supra- and infraliminal stimulation, ipsilateral stimulation, and stimulation on the opposite side of the tinnitus, in both single and repeat mode, were performed. The supraliminal stimulation on the opposite side of the tinnitus gave the best results. The best improvement appeared 12 hours after the stimulation and lasted 36 hours $(1 \mathrm{~Hz}, 120 \%$ of the opposite side motor threshold). A total of 1200 pulses were delivered. The TMS in single-pulse mode was ineffective on the patient's tinnitus.

\section{Functional MRI}

Functional MRI was realized before and 18 hours after TMS for two sessions. Five runs of acquisitions were performed on a Philips Medical Systems Achieva 3 Tesla (Philips Healthcare, Best, The Netherlands).

\section{Acquisition procedure}

The acquisition procedure was performed on a Philips Achieva 3 Tesla. For each session, an anatomic sequence was acquired, a T1-weighted 3D Turbo Field Echo sequence with the following parameters: the repetition time (TR): 9.35 milliseconds, echo time (TE): 4.6 milliseconds, angle flip 8 degrees, matrix: $256 \times 256,180$ slices, voxel size: $1 \times 1 \times 1 \mathrm{~mm}$.

The functional acquisition was performed with an echo planar imaging (EPI) sequence, fast field echo with a TR: 3000 milliseconds; TE: 33 milliseconds, reconstructed matrix size: $128 \times 128$, and voxel size: $1.88 \times 1.88 \times 4 \mathrm{~mm}, 34$ slices.

The paradigm was composed of nine blocks of 10 dynamics (five resting blocks, four activation blocks). The "activation" condition was a sound, without words, with multiple rhythms and including a wide range of frequencies. The "resting" condition was a period with no sound.

The patient was wearing a piezo ceramic cell headphone, which isolated her from the MRI noise but let her hear the stimulating sound.

The images were processed with SPM5 software (MATLAB, The MathWorks, Inc, Natick, MA). These images, without TMS, provided data about both sides of the auditory cortex. They confirmed the precise location of the auditory cortex.

The statistical maps of activation were obtained by using the following established procedures: slice timing correction, motion correction, and smooth spatially by using a 5-mm isotropic Gaussian kernel. The statistical analysis was performed with a statistical threshold at $P<.05$, corrected values and then merged on the anatomic image.

The results confirmed the theory about the auditory cortex asymmetry published in different articles ${ }^{3-7}$ and the hyperactivation of the primary and secondary auditory cortex on the left side (the patient is right handed). This is the opposite side of the tinnitus. 
Functional MRI after rTMS allowed comparing the different levels of cortical activation so we could evaluate the impact of the stimulation. Comparing the two sessions, we identified a predominant lessening on the auditory cortex of the left side (stimulation side). The refinement of the images allowed the objectification of the stimulation lessening, which touched the secondary auditory cortex and lowered the probability and volume of activation. The volume of activation areas of the left auditory cortex (I and II) was calculated before stimulation $\left(8.1 \mathrm{~cm}^{3}\right)$ and after stimulation $\left(4.9 \mathrm{~cm}^{3}\right)$ with the Medtronic Stealhstation (Medtronic Ltd, Hertfordshire, UK) (Figure 1).

\section{Surgical treatment}

\section{Preplanning}

Settings were programmed on the neuronavigation workstation (Stealhstation, Medtronic Ltd.). An anatomic MRI was merged with two fMRIs (before and after rTMS). We analyzed the primary and secondary auditory cortex in three dimensions, the areas activated or not by rTMS. We used the changes of blood-oxygen-level dependent (BOLD) response to rTMS as a means of refining the target, and also the anatomic criteria. With this information, the best target for the electrode plots was chosen. We decided to implant electrodes on both kinds of the auditory cortex, with the main part on the secondary auditory cortex (Figure 2).

The limit between the Heschl's gyri and the planum temporale was taken as a reference mark. We put one plot

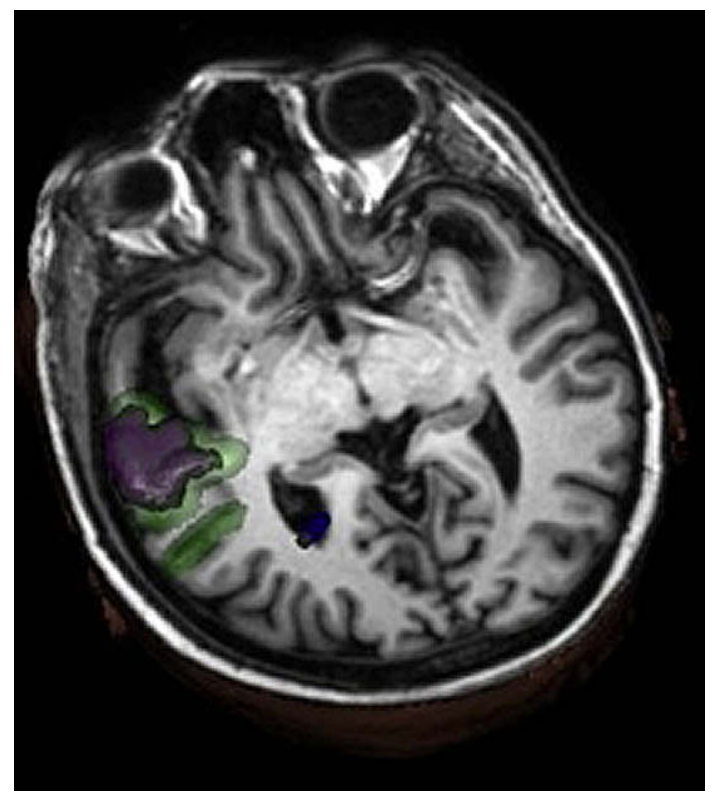

Figure 1 Variation of the activated auditory cortex before (green) and after (purple) rTMS.

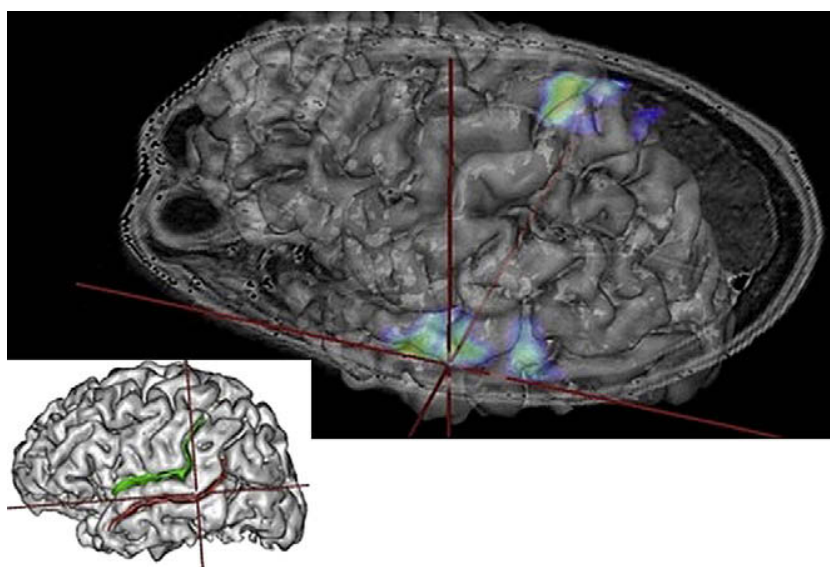

Figure 2 Representation of the ideal location of the plot 2 after we amalgamated the three dimensions. Imaging and datas of auditory cortex activated before rTMS.

on the primary auditory cortex and three plots on the secondary auditory cortex.

\section{Operation}

For a fast wakeup, we used propofol (diprivan) and remifentanil (ultiva) for the general anesthesia. Neuronavigation helped us to implant the two electrodes extradurally according to the spots defined during preplanning. An electroencephalogram (EEG) was performed using those two electrodes. We did recordings (during anesthesia and consciousness) according to the locations defined during preplanning, with and without auditory stimulation. We have recorded, on the electrode, the EEG in the auditory cortical surface. White noises at $90 \mathrm{~dB}$ of intensity, or the brief sound made by hitting a hammer on a metallic surface were used as auditory stimuli. The responses were better with some plots of the electrodes than with others. Results were much better with the patient awake (Table 1). These findings were in accordance with fMRI and the preplanning.

Plot 6 of the electrode II gave the best results. It was located on the secondary auditory cortex, the same location where the activity lowered after rTMS (Figure 3). The second part of the surgical procedure was performed 4 days later, a Synergy stimulating device (Medtronic Ltd.) was implanted under the skin (within the subclavicle area) and connected to the electrodes by two wires.

\section{Postplanning}

A computed tomogram (CT)-brain without injection after the first part of the procedure did not show any complications. Electrode locations were also checked. The CT was merged to the anatomic and fMRIs made before surgery (Figure 4) with the same workstation (Stealhstation, Medtronic, Ltd.). The images resulting from that confirmed the location of the electrodes in accordance with the locations defined during the functional investigations. 
Table 1 Results of electroencephalogram during surgery

\begin{tabular}{|c|c|c|c|c|c|}
\hline & \multirow[b]{2}{*}{ Plots } & \multicolumn{2}{|l|}{ White noise } & \multicolumn{2}{|l|}{ Brief sound } \\
\hline & & General anesthesia & Light anesthesia & General anesthesia & Light anesthesia \\
\hline \multirow[t]{3}{*}{ Electrode I } & 0 & No reply & No reply & No reply & No reply \\
\hline & 2 & No reply & No reply & Reply+ & Reply ++ \\
\hline & 3 & No reply & Reply+ & Reply ++ & Reply +++ \\
\hline \multirow[t]{3}{*}{ Electrode II } & 4 & Reply+ & Reply+ & Reply+ & Reply ++ \\
\hline & 6 & No reply & No reply & Reply ++ & Reply ++++ \\
\hline & 7 & No reply & No reply & No reply & No reply \\
\hline
\end{tabular}

We have identified the intensity of response from noise stimuli in each plot of the electrode.

\section{Follow-up}

The patient stayed 4 days in the neurosurgical unit after the second part of the procedure. On the second day the stimulating device was started. We used the main parameters published for tinnitus and chronic pain. Drouot et $\mathrm{al}^{8}$ suggested a sequential pattern between 1 and $3 \mathrm{~V}$ for the analgesic stimulation. De Ridder et $\mathrm{al}^{9}$ proposed continuous mode and long stimulation for the tinnitus treatment. The programming for the stimulation, at first, followed our experience in analgesic stimulation. The parameters were modified regularly during consultations until we found the right settings. We decided to start the stimulation with just one electrode, bipolar, and according to the data provided by the fMRI and the EEG. The plots 5 and 6 of the electrode II were chosen because they are closer to the cortex hypoactivated by rTMS and are the best compromise according to the functional and electrophysiologic data. The cathode is the plot 5 and the anode is the plot 6 .

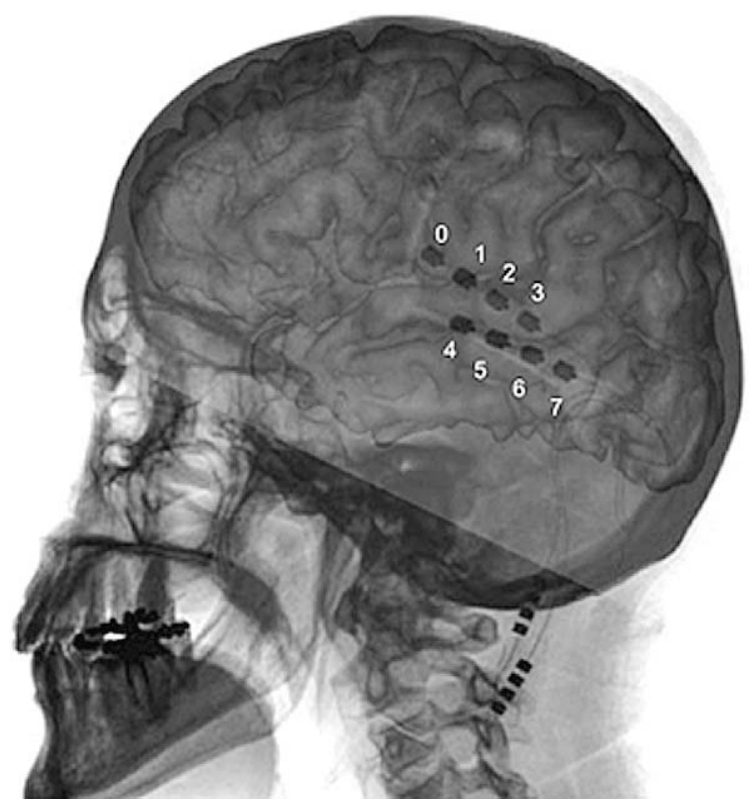

Figure 3 Fusion of the three dimension images of the left cerebral hemisphere and a profile radiography of the skull.

\section{First results}

The first responses were in the first 24 hours (18 hours). The patient replied to the TRQ questionnaire 10 days after starting the stimulating device and the final score was 58 , which meant a $25 \%$ lessening of the tinnitus impact.

The TRQ at 3 and 6 months were steady with an improvement of $65 \%$, and $80 \%$ at 1 year without any side effects.

\section{Discussion}

Tinnitus, often annoying, can become disabling. ${ }^{10}$ In such cases, a therapeutic approach must be adapted. The stimulation of the auditory cortex seems to be too immoderate. Functional changes that are caused by the expression of modulation of brain activity are best known in the somatosensory system, in which the role of neural plasticity in the cause of central neuropathic pain has been studied extensively. ${ }^{11,12}$

The auditory cortex is sophisticated with unclear boundaries. Our work, based on the data published in different articles, confirms the feasibility of the cortical stimulation with particular thanks given to the rTMS and fMRI. Smith et $\mathrm{al}^{3}$ made a map of the auditory pathways by using fMRI on patients who have tinnitus and in healthy self-willed people. They showed a lateralization of the tinnitus on the auditory pathway and in the cortex.

Eichhammer et $\mathrm{al}^{13}$ studied the auditory cortex with positron emission tomography (PET) scan with rTMS on three patients, the stimulation frequency was 2000 pulses at $1 \mathrm{~Hz}$. The tinnitus was reduced on the opposite side of the stimulation.

Kleinjung et $\mathrm{al}^{6}$ included 14 patients with disabling tinnitus in their study, in which the auditory cortex was located by PET scan. The results were in accordance with the other recent publication results.

In 2006, Londero et $\mathrm{al}^{14}$ presented a study with 13 patients treated by rTMS, in supraliminal mode, 1200 pulses at $1 \mathrm{~Hz}$. The stimulation was performed according to data from fMRI. The results were significant. 

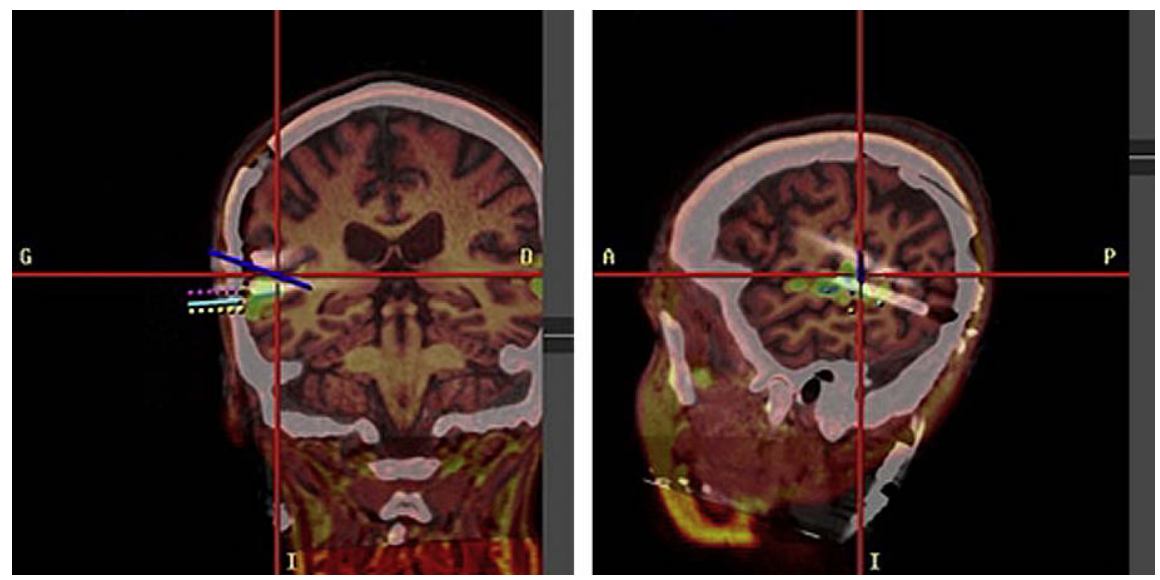

Figure 4 Postplanning, showing the fusion of anatomic and functional magnetic resonance imaging and computed tomographic brain after surgery. We can check the location of the electrodes put in place on the areas where the cortex is activated.

So, the TMS on the auditory cortex opposite to the tinnitus side provided a significant but transitory lessening of the symptom. The mechanism of neuromodulation is still unsure. The review made by Londero et $\mathrm{al}^{15}$ showed the inhomogenicity of the different results and the difficulty with extracting solid conclusions. However, cortical neuromodulation with thalamic projections seems to be clear. The best results were obtained with a low-stimulation frequency $(1 \mathrm{~Hz})$, but the right frequency of stimulation needs to be determined.

The TMS gives a temporary effect on the tinnitus. The functional study is indispensable in the selection of the patients. The predictive characteristic of the TMS in the answer has not been proved yet, but it is used by a lot of teams.

Functional MRI characterizes the organic feature of the tinnitus. According to our first results, it shows the neuromodulation by the rTMS. The data in the literature about electrical stimulation in tinnitus treatment is very rare, only two teams published results on the subject. De Ridder et $\mathrm{al}^{16}$ studied 12 patients with tinnitus, treated by cortical stimulation (10 patients with unilateral tinnitus, two with bilateral tinnitus). The results were promising, especially in unilateral cases with narrow frequency. There was a lessening of symptoms in $97 \%$ of the patients with a score of 0.5 of 10 on a VAS (1.5 before). Tinnitus with a large range of frequency presented a lessening of the symptoms in only $24 \%$ of the patients and a score of 6.8 of 10 on a VAS ( 8.8 before).

The authors insist on the importance of acouphenometry in determining the range of frequency of the tinnitus. The adjustment of the stimulation is cyclical, which seems important, and the possibility of a remote control for the patients was expressed so they could modify the settings at any time.

Friedland et $\mathrm{al}^{17}$ studied the feasibility of auditory cortical stimulation for the treatment of tinnitus. Their study included eight patients with unilateral tinnitus and a surgical implantation of an epidural electrode that used fMRI targeting. They showed that chronic stimulation of the secondary auditory cortex seems to be a potential therapeutic treatment for the suppression of tinnitus.

\section{Conclusion}

The success of the surgery and the first results encourage us to pursue our study. The entire procedure has just been established within these last 2 years. We have to keep in mind only $10 \%$ of the patients seen for tinnitus can be candidates for the protocol. The other $90 \%$ cannot because they do not correspond to our criteria, or do not want to participate because of the length and involvement of the procedure.

\section{References}

1. Giraud AL, Chery-Croze S, Fischer G, et al. A selective imaging of tinnitus. Neuroreport 1999;10(1):1-5.

2. Wilson PH, Henry J, Bowen M, Haralambous G. Tinnitus reaction questionnaire: psychometric properties of a measure of distress associated with tinnitus. J Speech Hear Res 1991;34(1):197-201.

3. Smith JA, Mennemeier M, Bartel T, et al. Repetitive transcranial magnetic stimulation for tinnitus: a pilot study. Laryngoscope 2007;117(3):529-534.

4. Wang H, Tian J, Yin D, et al. Regional glucose metabolic increases in left auditory cortex in tinnitus patients: a preliminary study with positron emission tomography. Chin Med J (Engl) 2001;114(8):848-851.

5. Liegeois-Chauvel C, Giraud K, Badier JM, Marquis P, Chauvel P. Intracerebral evoked potentials in pitch perception reveal a functional asymmetry of the human auditory cortex. Ann N Y Acad Sci 2001;930:117-132.

6. Kleinjung T, Steffens T, Langguth B, et al. [Treatment of chronic tinnitus with neuronavigated repetitive transcranial magnetic stimulation (rTMS).]. Hno 2006;54(6):439-444.

7. Langguth B, Eichhammer P, Zowe M, et al. [Low frequency repetitive transcranial magnetic stimulation (rTMS) for the treatment of chronic tinnitus-are there long-term effects?]. Psychiatr Prax 2004;31(Suppl 1): S52-S54.

8. Drouot X, Nguyen JP, Peschanski M, Lefaucheur JP. The antalgic efficacy of chronic motor cortex stimulation is related to sensory changes in the painful zone. Brain 2002;125(Pt 7):1660-1664.

9. De Ridder D, De Mulder G, Walsh V, Muggleton N, Sunaert S, Moller A. Magnetic and electrical stimulation of the auditory cortex for intractable tinnitus: case report. J Neurosurg 2004;100(3):560-564.

10. Heller AJ. Classification and epidemiology of tinnitus. Otolaryngol Clin North Am 2003;36(2):239-248.

11. Flor H, Elbert T, Muhlnickel W, Pantev C, Wienbruch C, Taub E. Cortical reorganization and phantom phenomena in congenital and traumatic upper-extremity amputees. Exp Brain Res 1998;119(2): 205-212. 
12. Lockwood AH, Salvi RJ, Burkard RF, Galantowicz PJ, Coad ML, Wack DS. Neuroanatomy of tinnitus. Scand Audiol (Suppl) 1999;51: 47-52.

13. Eichhammer P, Langguth B, Marienhagen J, Kleinjung T, Hajak G. Neuronavigated repetitive transcranial magnetic stimulation in patients with tinnitus: a short case series. Biol Psychiatry 2003; 54(8):862-865.

14. Londero A, Lefaucheur JP, Malinvaud D, et al. [Magnetic stimulation of the auditory cortex for disabling tinnitus: preliminary results]. Presse Med 2006;35(2 Pt 1):200-206.
15. Londero A, Langguth B, De Ridder D, Bonfils P, Lefaucheur JP. Repetitive transcranial magnetic stimulation (rTMS): a new therapeutic approach in subjective tinnitus? Neurophysiol Clin 2006; 36(3):145-155.

16. De Ridder D, De Mulder G, Verstraeten E, et al. Primary and secondary auditory cortex stimulation for intractable tinnitus. ORL J Otorhinolaryngol Relat Spec 2006;68(1):48-54. discussion: 5.

17. Friedland DR, Gaggl W, Runge-Samuelson C, Ulmer JL, Kopell BH. Feasibility of auditory cortical stimulation for the treatment of tinnitus. Otol Neurotol 2007;28(8):1005-1012. 